\title{
Cluster identification, selection, and description in cluster randomized crossover trials: the PREP-IT trials
}

Sheila Sprague ${ }^{1,2^{*}} \mathbb{D}$, Taryn Scott ${ }^{1}$, Shannon Dodds ${ }^{1}$, David Pogorzelski ${ }^{1}$, Paula McKay ${ }^{1}$, Anthony D. Harris ${ }^{3}$, Amber Wood ${ }^{4}$, Lehana Thabane ${ }^{2}$, Mohit Bhandari ${ }^{1,2}$, Samir Mehta ${ }^{5}$, Greg Gaski $^{6}$, Christina Boulton", Francesc Marcano-Fernández ${ }^{8}$, Ernesto Guerra-Farfán ${ }^{9}$, Joan Hebden ${ }^{10}$, Lyndsay M. O'Hara ${ }^{3}$, Gerard P. Slobogean ${ }^{11}$, on behalf of the PREP-IT Investigators

\begin{abstract}
Background: In cluster randomized crossover (CRXO) trials, groups of participants (i.e., clusters) are randomly allocated to receive a sequence of interventions over time (i.e., cluster periods). CRXO trials are becoming more comment when they are feasible, as they require fewer clusters than parallel group cluster randomized trials. However, CRXO trials have not been frequently used in orthopedic fracture trials and represent a novel methodological application within the field. To disseminate the early knowledge gained from our experience initiating two cluster randomized crossover trials, we describe our process for the identification and selection of the orthopedic practices (i.e., clusters) participating in the PREP-IT program and present data to describe their key characteristics.

Methods: The PREP-IT program comprises two ongoing pragmatic cluster randomized crossover trials (AqueousPREP and PREPARE) which compare the effect of iodophor versus chlorhexidine solutions on surgical site infection and unplanned fracture-related reoperations in patients undergoing operative fracture management. We describe the process we used to identify and select orthopedic practices (clusters) for the PREP-IT trials, along with their characteristics.

\footnotetext{
* Correspondence: sprags@mcmaster.ca

${ }^{1}$ Department of Surgery, Division of Orthopaedic Surgery, McMaster

University, 293 Wellington Street North, Suite 110, Hamilton, ON L8L 8E7, Canada

${ }^{2}$ Department of Health Research Methods, Evidence, and Impact, McMaster University, 293 Wellington St. N., Suite 110, Hamilton, ON L8L 8E7, Canada Full list of author information is available at the end of the article
}

(c) The Author(s). 2020 Open Access This article is licensed under a Creative Commons Attribution 4.0 International License, which permits use, sharing, adaptation, distribution and reproduction in any medium or format, as long as you give appropriate credit to the original author(s) and the source, provide a link to the Creative Commons licence, and indicate if changes were made. The images or other third party material in this article are included in the article's Creative Commons licence, unless indicated otherwise in a credit line to the material. If material is not included in the article's Creative Commons licence and your intended use is not permitted by statutory regulation or exceeds the permitted use, you will need to obtain permission directly from the copyright holder. To view a copy of this licence, visit http://creativecommons.org/licenses/by/4.0/. The Creative Commons Public Domain Dedication waiver (http://creativecommons.org/publicdomain/zero/1.0/) applies to the data made available in this article, unless otherwise stated in a credit line to the data. 


\begin{abstract}
(Continued from previous page)
Results: We identified 58 potential orthopedic practices for inclusion in the PREP-IT trials. After screening each practice for eligibility, we selected 30 practices for participation and randomized each to a sequence of interventions (15 for Aqueous-PREP and 20 for PREPARE). The majority of orthopedic practices included in the Aqueous-PREP and PREPARE trials were situated in level I trauma centers (100\% and 87\%, respectively). Orthopedic practices in the Aqueous-PREP trial operatively treated a median of 149 open fracture patients per year, included a median of 11 orthopedic surgeons, and had access to a median of 5 infection preventionists. Orthopedic practices in the PREPARE trial treated a median of 142 open fracture and 1090 closed fracture patients per year, included a median of 7.5 orthopedic surgeons, and had access to a median of 6 infection preventionists.
\end{abstract}

Conclusions: The PREP-IT trials provide an example of how to follow the reporting standards for cluster randomized crossover trials by providing a clear definition of the cluster unit, a thorough description of the cluster identification and selection process, and sufficient description of key cluster characteristics.

Trial registration: Both trials are registered at ClinicalTrials.gov (A-PREP: NCT03385304 December 28, 2017, and PREPARE: NCT03523962 May 14, 2018).

Keywords: Cluster, Randomized crossover, Pragmatic, Cluster characteristics, Orthopedic, Surgical site infection

\section{Background}

In clinical research, large high-quality randomized controlled trials (RCTs) are considered the highest level of evidence to determine the effectiveness of an intervention [1]. While most RCTs follow a parallel group design and randomize individual participants to one or more intervention and control groups, there are certain situations in which randomization at this level is not feasible or practical [2]. Cluster randomized trials overcome these challenges by randomizing predetermined groups of participants (i.e., clusters) to interventions [3]. In cluster randomized crossover (CRXO) trials, each cluster serves as their own control group by participating in both the treatment and control arm at least once at various periods of time throughout the trial. An advantage to this design [CRXO] over the parallel group cluster randomized trial is that fewer clusters are required to achieve statistical power.

Researchers undertaking CRXO trials must ensure that they take into consideration multiple differences in the conduct and reporting between RCTs and CRXO trials $[4,5]$. One of these fundamental differences is the requirement to provide information on the characteristics and flow of clusters throughout the conduct of a CRXO trial (i.e., enrolment, allocation, follow-up, analysis), as described in the CONSORT extension for cluster randomized trials [4]. This is accomplished by including a priori cluster selection criteria (similar to participant eligibility criteria) and a cluster flow diagram that shows the cluster selection and flow over the duration of the trial (similar to the patient flow diagram). This information enables knowledge users to assess the generalizability of the trial and determine if the results are applicable to their setting and practice.

To disseminate the early knowledge gained from our experience initiating two large infection prevention CRXO trials in orthopedic fracture patients, we describe our cluster identification and selection process. Additionally, we present data on the key characteristics of the clusters to illustrate the level of data collection that is required for appropriate reporting in CRXO trials.

\section{Methods \\ The PREP-IT program}

The Program of Randomized trials to Evaluate Preoperative antiseptic skin solutions in orthopaedic Trauma (PREP-IT) aims to determine the effectiveness of iodophor compared to chlorhexidine solutions at reducing surgical site infection (SSI) and unplanned fracture-related reoperations in fracture surgery patients. The PREP-IT program includes two ongoing multi-center pragmatic CRXO trials that study the effects of four antiseptic solutions in three independent populations of surgically treated fracture patients: the Aqueous-PREP (A Pragmatic Randomized trial Evaluating Pre-operative aqueous antiseptic skin solutions in open fractures) trial and the PREPARE (A Pragmatic Randomized trial Evaluating Pre-operative Alcohol skin solutions in Fractured Extremities) trial (Fig. 1). The Aqueous-PREP trial compares $4 \%$ aqueous chlorhexidine versus $10 \%$ povidone-iodine in open extremity fracture patients. The PREPARE trial compares $2 \%$ chlorhexidine in $70 \%$ isopropyl alcohol (ChloraPrep ${ }^{\mathrm{TM}}$ ) versus $0.7 \%$ iodine povacrylex in $74 \%$ isopropyl alcohol (DuraPrep ${ }^{\mathrm{Tw}}$ ) in both open extremity fracture patients and patients with closed lower extremity or pelvic fractures. Both trials follow a single master protocol which has been described in a previous manuscript [6].

Briefly, the order of treatment allocation for each orthopedic practice (i.e., cluster) was randomly assigned using a computer-generated randomization table. Simple, non-stratified randomization was used. Cluster randomization is performed centrally at the Methods Centre by personnel who are unaware of the cluster 


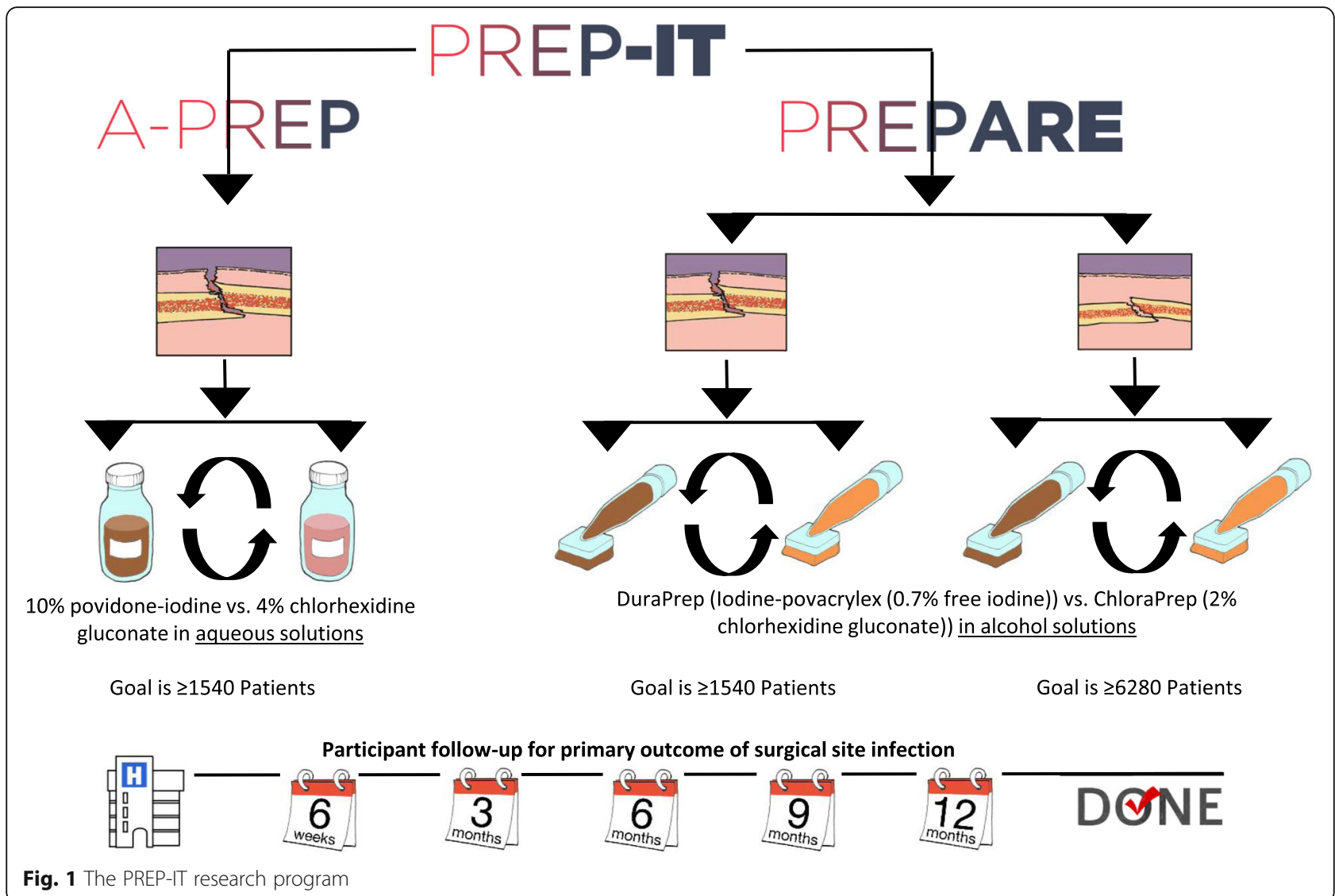

characteristics. Randomization is performed when a cluster has received local ethics approval and their clinical trial agreement is in place.

Each orthopedic practice starts with the surgical preparation solution to which they were initially allocated and subsequently crosses over to the other solution for their second cluster period. This process of alternating treatments repeats approximately every 2 months as dictated by the initial randomization. This process of alternating treatment periods (crossovers) will continue until the minimum sample size is achieved for each fracture population and the study's planned recruitment period is completed. Clinical sites must complete at least two treatment periods (one crossover), and the majority of clinical sites will complete 12 treatment periods (11 crossovers), and 24 months of enrollment.

For both trials, the primary outcome is SSI as defined by the Centers for Disease Control and Prevention (CDC) [7] which includes superficial incisional SSI within 30 days and deep incisional or organ/space SSI within 90 days of definitive fracture management surgery. We continue to follow participants for SSI for 12 months to inform our sensitivity analyses. The secondary outcome is unplanned fracture-related reoperations within 12 months to manage infection, wound healing problems, and fracture healing problems. The Aqueous-PREP trial will enroll a minimum of 1540 open fracture patients, and PREPARE will enroll a minimum of 1540 open fracture patients and 6280 closed fracture patients.

\section{Cluster definition}

We defined clusters as orthopedic practices within participating hospitals. Each participating hospital has only one participating orthopedic practice. Clustering occurs at the level of the orthopedic practice as only orthopedic surgeons were required to follow the study protocol and use the randomized surgical preparation solution. During the design phase, we considered defining clusters as either the hospitals with which orthopedic practices were affiliated or the operating rooms used by orthopedic practices; however, we decided against these definitions as other specialities associated with the hospital or operating rooms were not required to follow the study protocol.

\section{Cluster eligibility criteria}

To be eligible for inclusion in the PREP-IT trials, orthopedic practices were required to meet the predetermined 
eligibility criteria as specified in the trial protocols. Specifically, the inclusion criteria were as follows: (1) adequate research personnel infrastructure to manage the study, (2) adequate open fracture volume and closed lower extremity and pelvic fracture volume to complete enrollment within the study timeline (i.e., a minimum of 77 open fractures (for Aqueous-PREP and PREPARE) and 314 closed lower extremity fractures per year (for PREPARE only)), (3) commitment from all or most orthopedic surgeons to participate in the trial, and (4) ability to use the two skin preparation solutions. The exclusion criteria were as follows: (1) lack of interest in the trial; (2) anticipated challenges with complying with the protocol; (3) conflicting studies, in the judgment of the Principal Investigators, that would inhibit patient participation; (4) budgeting or contract constraints; and (5) conflicting roles within the trials (e.g., member of the Data and Safety Monitoring Board).

\section{Cluster identification and selection}

We identified orthopedic practices through professional contacts with the PREP-IT Principal Investigators. Each orthopedic practice received an email containing information about the PREP-IT trials, as well as an invitation to apply to participate in either one or both trials. For orthopedic practices who declined this invitation, we identified and documented the reason for exclusion using our predetermined eligibility criteria. Orthopedic practices who expressed a desire to participate were required to complete a feasibility questionnaire. This questionnaire consisted of 17 questions and assessed the orthopedic practice's research experience and infrastructure, fracture volume, current practice patterns, and interest in participating in one or both trials. We used the responses to the feasibility questionnaire to preliminarily screen orthopedic practices for eligibility. Orthopedic practices that were found to be ineligible through this preliminary screen were notified, and the reason for exclusion was documented. Orthopedic practices that were eligible to participate following the preliminary screen were invited to participate in a series of teleconferences with the PREP-IT Principal Investigators and study personnel. The purpose of these calls was to review trial and clinical practice logistics in detail and to confirm whether each orthopedic practice met the eligibility criteria for participation. For any orthopedic practice that was determined to be ineligible through these calls, we documented the reason for exclusion. Orthopedic practices that met all eligibility criteria were selected to participate.

\section{Collection of cluster characteristics}

Upon selection, orthopedic practices were required to complete a cluster definition questionnaire. This questionnaire, which is comprised of 44 questions, captures important data on hospital characteristics, current surgeon preferences and practices for pre-operative surgical preparation techniques, and infection co-interventions known to reduce the incidence of SSIs. Each orthopedic practice's questionnaire data is updated every 4 months over the enrollment and follow-up periods.

\section{Statistical analyses}

Our statistical analysis plan was determined a priori. We included data from the orthopedic practices currently enrolling participants into the Aqueous-PREP trial and the PREPARE trial. We used descriptive statistics to summarize all characteristics of the orthopedic practices (frequencies and percentages for categorical variables, and medians and interquartile ranges (IQRs) for continuous variables). We used Microsoft Excel 2016 to conduct all statistical analyses.

\section{Results}

\section{Cluster selection}

Of the 58 potentially eligible orthopedic practices that we identified, 47 (81\%) accepted our invitation to complete a feasibility questionnaire and 11 (19\%) declined our invitation and were excluded due to a lack of interest in the trial (Figs. 2 and 3). Of the 47 practices that completed the feasibility questionnaire, 12 were determined to be ineligible and 35 were invited to participate in a teleconference with the Principal Investigators and study team to further assess eligibility. Of the 35 orthopedic practices that participated in the teleconferences, 5 were determined to be ineligible, 5 were selected to participate in both the Aqueous-PREP and PREPARE trials, 10 in the Aqueous-PREP trial only, and 15 in the PREPARE trial only. Specific reasons for orthopedic practice exclusions for each trial are documented in Figs. 2 and 3. One of the practices participating in the Aqueous-PREP trial was discontinued post-randomization due to an inability to follow the trial protocol. Specifically, this cluster did not meet the a priori threshold of treatment compliance (e.g., 90\% of patients receive the allocated antiseptic solution) and the clinical site was unable to complete the case report forms, which led to incomplete data submission. The clinical site enrolled 14 participants and was withdrawn within 2 months of initiation. Therefore, there are 14 orthopedic practices in the Aqueous-PREP trial and 20 orthopedic practices in the PREPARE trial.

\section{Characteristics of participating clusters}

Table 1 details the name and location of the hospital associated with each orthopedic practice participating in the Aqueous-PREP and PREPARE trials, as well as the intervention sequence to which they were randomized. 


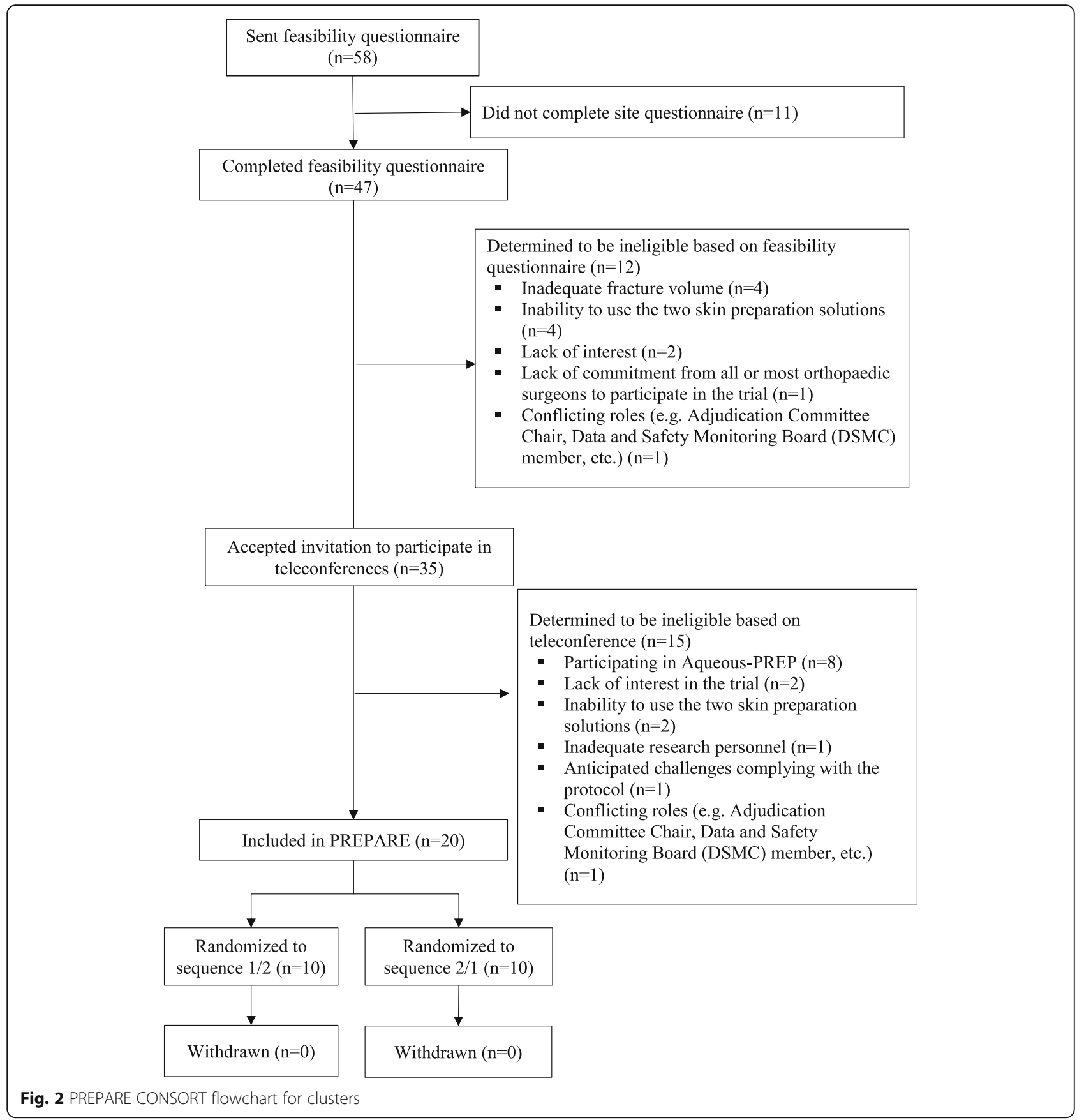

The majority of orthopedic practices are affiliated with hospitals that are level I trauma centers and owned by a non-profit, non-religious organization (Table 2). The median population served is $1,625,000$ for orthopedic practices participating in the Aqueous-PREP trial and 2, 400,000 for the PREPARE trial. The characteristics of the withdrawn cluster are presented in the Appendix.

Orthopedic practices participating in the AqueousPREP trial treat a median of 149 open fractures annually and include a median of 11 orthopedic surgeons who treat fractures and 8.5 who take trauma calls (Table 3 ).
Orthopedic practices participating in the PREPARE trial treat a median of 142 open fractures annually and 1090 closed fractures annually. These orthopedic practices include a median of 7.5 orthopedic surgeons who treat fracture patients and 9.5 who take trauma calls.

Participating orthopedic practices employ a variety of surgical infection prevention measures (Table 4). Specifically, practices participating in the Aqueous-PREP trial have a median of 2.2 infection preventionists per 250 hospital beds. Sixty-four percent of hospitals $(n=9)$ perform active surveillance for methicillin-resistant 


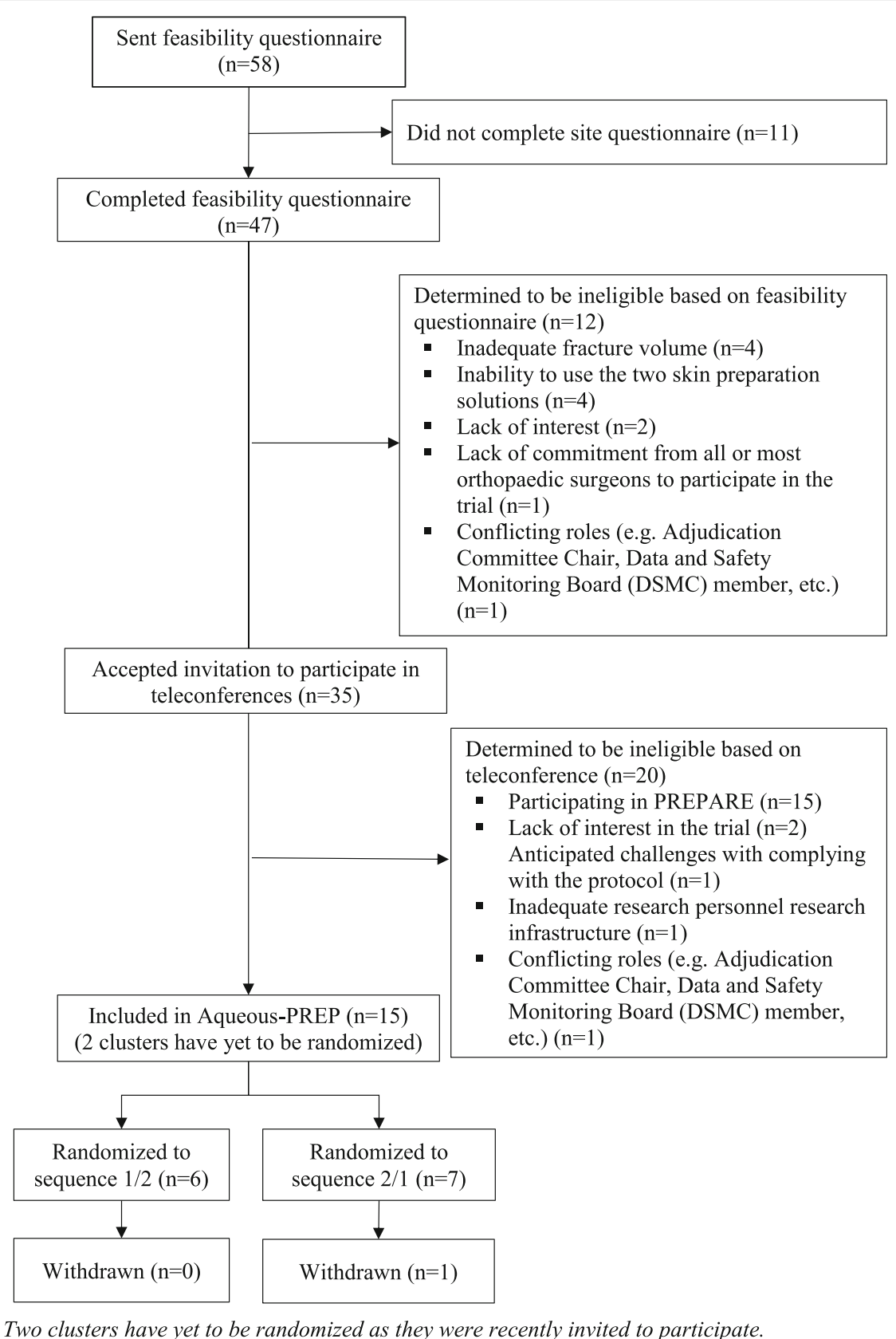

Note: Two clusters have yet to be randomized as they were recently invited to participate.

Fig. 3 Aqueous-PREP CONSORT flowchart for clusters

Staphylococcus aureus (MRSA) in open fracture patients. None of the clinical sites perform active surveillance for vancomycin-resistant Enterococcus (VRE) in open fracture patients. Sixty-four percent of practices $(n=9)$ have a decolonization protocol for MRSA. All practices have some infection control parameters in place in their operating rooms (e.g., high-efficiency particulate air (HEPA) filters, positive pressure operating rooms, air changes per hour, temperature maintained between 20 and $24^{\circ} \mathrm{C}$, and humidity between 20 and $60 \%$ ).
Orthopedic practices participating in the PREPARE trial employ a median of 2.6 infection preventionists per 250 hospital beds. Twenty percent of orthopedic practices $(n=4)$ perform active surveillance for MRSA in open fracture patients, and $15 \%$ of orthopedic practices $(n=3)$ perform active surveillance in closed fracture patients. No clusters perform active surveillance for VRE in either open or closed fracture patients. Sixty percent $(n=12)$ of orthopedic practices have a decolonization protocol for MRSA. Additionally, all practices had some 
Table 1 Clusters and location

\begin{tabular}{|c|c|c|c|}
\hline Hospital (cluster) name & Location & $\begin{array}{l}\text { Randomization sequence } \\
(1 / 2 \text { vs. } 2 / 1)\end{array}$ & Trial \\
\hline $\begin{array}{l}\text { University of Maryland School of Medicine, R Adams Cowley Shock } \\
\text { Trauma Center }\end{array}$ & Baltimore, MD & $\begin{array}{l}\text { 2/1 (Aqueous-PREP); } 1 / 2 \\
\text { (PREPARE) }\end{array}$ & $\begin{array}{l}\text { Aqueous-PREP and PREP } \\
\text { ARE }\end{array}$ \\
\hline Greenville Health System & Greenville, SC & $\begin{array}{l}\text { 2/1 (Aqueous-PREP); 2/1 } \\
\text { (PREPARE) }\end{array}$ & $\begin{array}{l}\text { Aqueous-PREP and PREP } \\
\text { ARE }\end{array}$ \\
\hline Hamilton Health Sciences - General Site & Hamilton, ON & $\begin{array}{l}\text { 1/2 (Aqueous-PREP); } 2 / 1 \\
\text { (PREPARE) }\end{array}$ & $\begin{array}{l}\text { Aqueous-PREP and PREP } \\
\text { ARE }\end{array}$ \\
\hline IU Health Methodist Hospital & Indianapolis, IN & $\begin{array}{l}1 / 2 \text { (Aqueous-PREP); } 1 / 2 \\
\text { (PREPARE) }\end{array}$ & $\begin{array}{l}\text { Aqueous-PREP and PREP } \\
\text { ARE }\end{array}$ \\
\hline San Antonio Military Medical Center & San Antonio, TX & $\begin{array}{l}1 / 2 \text { Aqueous-PREP; } 1 / 2 \\
\text { (PREPARE) }\end{array}$ & $\begin{array}{l}\text { Aqueous-PREP and PREP } \\
\text { ARE }\end{array}$ \\
\hline Banner - University Medical Center Tucson & Tucson, AZ & $1 / 2$ & Aqueous-PREP \\
\hline Hospital Universitari Parc Tauli & Barcelona, Spain & To be randomized & Aqueous-PREP \\
\hline McGovern Medical School at UTHealth Houston & Houston, TX & $1 / 2$ & Aqueous-PREP \\
\hline The CORE Institute & Phoenix, AZ & $2 / 1$ & Aqueous-PREP \\
\hline University of California, San Francisco & San Francisco, CA & $1 / 2$ & Aqueous-PREP \\
\hline University of Florida & Gainesville, FL & $2 / 1$ & Aqueous-PREP \\
\hline Vall d'Hebron Hospital & Barcelona, Spain & To be randomized & Aqueous-PREP \\
\hline Vanderbilt Medical Center & Nashville, TN & $2 / 1$ & Aqueous-PREP \\
\hline Wright State University & Dayton, $\mathrm{OH}$ & $2 / 1$ & Aqueous-PREP \\
\hline Brigham and Women's Hospital & Boston, MA & $1 / 2$ & PREPARE \\
\hline Carolina's Medical Center & Charlotte, NC & $2 / 1$ & PREPARE \\
\hline Dartmouth-Hitchcock Medical Center & Lebanon, NH & $2 / 1$ & PREPARE \\
\hline Duke University Hospital & Durham, NC & $2 / 1$ & PREPARE \\
\hline Inova Fairfax Medical Campus & Fairfax, VA & $2 / 1$ & PREPARE \\
\hline Massachusetts General Hospital & Boston, MA & $1 / 2$ & PREPARE \\
\hline MetroHealth Medical Center & Cleveland, $\mathrm{OH}$ & $1 / 2$ & PREPARE \\
\hline Royal Columbian Hospital & $\begin{array}{l}\text { New Westminster, } \\
\text { BC }\end{array}$ & $2 / 1$ & PREPARE \\
\hline Regional Medical Center of San Jose & San Jose, CA & $1 / 2$ & PREPARE \\
\hline Sanford Health & Sioux Falls, SD & $1 / 2$ & PREPARE \\
\hline University of Maryland Capital Regional Health & Cheverly, MD & $2 / 1$ & PREPARE \\
\hline University of Mississippi Medical Center & Jackson, MS & $2 / 1$ & PREPARE \\
\hline University of Pennsylvania & Philadelphia, PA & $1 / 2$ & PREPARE \\
\hline University of Utah & Salt Lake City, UT & $2 / 1$ & PREPARE \\
\hline Wake Forest Baptist Hospital & $\begin{array}{l}\text { Winston-Salem, } \\
\text { NC }\end{array}$ & $1 / 2$ & PREPARE \\
\hline
\end{tabular}

infection control parameters in place in their operating rooms.

\section{Discussion}

The Aqueous-PREP and PREPARE trials represent two ongoing CRXO trials within the field of orthopedics. The successes we have experienced with cluster identification and selection provide support for the feasibility of using this trial design in orthopedic trauma trials. As part of the PREP-IT program's commitment to transparency and upholding rigorous reporting standards, this paper clearly defines the cluster unit, provides detailed information about the cluster identification and selection process, and describes key characteristics of the included clusters (Fig. 4). These are some of the key reporting requirements described in the CONSORT extension for CRTs [4].

Given that the Aqueous-PREP and PREPARE trials use a pragmatic design, when selecting orthopedic practices (i.e., clusters), we aimed to include a sample that was 
Table 2 Cluster characteristics

\begin{tabular}{|c|c|c|}
\hline Characteristic & Aqueous-PREP, $\boldsymbol{N}=14^{*}$ & PREPARE, $\boldsymbol{N}=20$ \\
\hline \multicolumn{3}{|l|}{ Level of trauma care provided, $n(\%)$} \\
\hline Level I & $14(100)$ & $17(85)$ \\
\hline Level II & $0(0)$ & $3(15)$ \\
\hline \multicolumn{3}{|l|}{ Ownership of hospital, $n$ (\%) } \\
\hline Non-profit, not religious order affiliated & $8(57)$ & $15(75)$ \\
\hline Government & $4(29)$ & $4(20)$ \\
\hline Private & $1(7)$ & $1(5)$ \\
\hline Private administration with public funding & $1(7)$ & \\
\hline \multicolumn{3}{|l|}{ Hospital affiliation, n (\%) } \\
\hline Independent, free-standing & $2(14)$ & $1(5)$ \\
\hline Multi-facility organization (chain) & $5(36)$ & $5(25)$ \\
\hline Hospital system, attached & $4(29)$ & $7(35)$ \\
\hline Hospital system, free-standing & $3(21)$ & $7(35)$ \\
\hline Population size served, median (IQR) & $1,625,000(3,735,699)$ & $2,400,000(2,875,319)$ \\
\hline Number of inpatient beds, median (IQR) & $679(287.3)$ & $616(388.5)$ \\
\hline Number of operating rooms, median (IQR) & $21(18)$ & $31(22.5)$ \\
\hline Number of beds in intensive care unit, median (IQR) & $48.5(44.8)$ & $74(81.5)$ \\
\hline Hospital is a primary teaching hospital for a medical school, $n(\%)$ & $14(100)$ & $16(80)$ \\
\hline
\end{tabular}

$I Q R$ interquartile range

*The cluster that was withdrawn is not included

representative of the orthopedic practices that treat the patient population in "real world" usual care settings. This is an essential component of pragmatic trials which aim to determine how well interventions work in the "real world" as opposed to explanatory trials which aim to determine how well interventions can work in ideal settings [8]. To accomplish this goal, we designed our cluster eligibility criteria to be as broad as possible while still ensuring that participating orthopedic practices would have the infrastructure and patient volume to successfully follow the protocol and meet study timelines. Through our cluster selection process, we identified 58 potentially eligible orthopedic practices and ultimately selected 5 sites to participate in both the Aqueous-PREP and PREPARE trials, 10 in the AqueousPREP trial only, and 15 in the PREPARE trial only. This

Table 3 Orthopedic characteristics

\begin{tabular}{lll}
\hline Characteristic & Aqueous-PREP, $\boldsymbol{N}=14^{*}$ & PREPARE, $\boldsymbol{N}=20$ \\
\hline Number of orthopedic surgeons who treat fracture patients, median (IQR) & $11(11.3)$ & $7.5(7)$ \\
Number of orthopedic surgeons who take trauma calls, median (IQR) & $8.5(13)$ & $9.5(9.5)$ \\
Orthopedic training programs, $n(\%)^{* *}$ & $13(93)$ & $17(85)$ \\
Orthopedic fellowship & $14(100)$ & $18(90)$ \\
Orthopedic residency & $14(100)$ & $17(85)$ \\
Orthopedic clerkship & $0(0)$ & $1(5)$ \\
None of above & $149(125)$ & $142(185)$ \\
Annual number of operatively managed open fractures, median (IQR) & $1200(828.8)$ & $1090(630.5)$ \\
Annual number of operatively managed closed fractures, median (IQR) & - & $725(435)$ \\
Annual number of operatively managed closed lower extremity and pelvic fractures, median (IQR) & $4(2)$ & $4(5.5)$ \\
Number of operating rooms used to treat fractures, median (IQR) &
\end{tabular}

$I Q R$ interquartile range

*The cluster that was withdrawn is not included

**Percentages do not sum to 100 as categories are not mutually exclusive 
Table 4 Surgical infection prevention information

\begin{tabular}{|c|c|c|}
\hline Characteristic & Aqueous-PREP, $\boldsymbol{N}=14^{*}$ & PREPARE, $\boldsymbol{N}=20$ \\
\hline Number of infection preventionists per 250 hospital beds, median (IQR) & $2.2(1.7)$ & $2.6(1.9)$ \\
\hline Infection preventionists certified in infection control, median (IQR) & $3(2.8)$ & $4(3)$ \\
\hline Perform active surveillance cultures for MRSA in open fracture patients, $n(\%)$ & $9(64)$ & $4(20)$ \\
\hline Perform active surveillance cultures for VRE in open fracture patients, $n$ (\%) & $0(0)$ & $4(20)$ \\
\hline Perform active surveillance cultures for other organisms in patients with open fractures, $n(\%)$ & $2(14)$ & $3(15)$ \\
\hline Perform active surveillance cultures for MRSA in closed fracture patients, $n$ (\%) & - & $3(15)$ \\
\hline Perform active surveillance cultures for VRE in closed fracture patients, $n(\%)$ & - & $3(15)$ \\
\hline Perform active surveillance cultures for other organisms in patients with closed fractures, $n$ (\%) & - & $1(5)$ \\
\hline Decolonization protocol for MRSA, $n(\%)$ & $9(64)$ & $12(60)$ \\
\hline Policies and/or guidelines on maintaining normothermia during perioperative period, $n$ (\%) & $14(100)$ & $16(80)$ \\
\hline \multicolumn{3}{|l|}{ Airflow system(s) in operating room, $n(\%)^{* *}$} \\
\hline None & $0(0)$ & $1(5)$ \\
\hline Vertical laminar flow & $9(64)$ & $14(70)$ \\
\hline Conventional ventilation & $6(43)$ & $7(35)$ \\
\hline Other & $0(0)$ & \\
\hline \multicolumn{3}{|l|}{ Parameters in place in hospital operating rooms, $n(\%)^{* *}$} \\
\hline High-efficiency particulate air (HEPA) filters & $12(86)$ & $15(75)$ \\
\hline Operating rooms are positive pressure & $14(100)$ & $16(80)$ \\
\hline Air changes per hour & $13(93)$ & $17(85)$ \\
\hline Temperature maintained between 20 and $24^{\circ} \mathrm{C}$ and humidity between 20 and $60 \%$ & $13(93)$ & $18(90)$ \\
\hline
\end{tabular}

*The cluster that was withdrawn is not included

**Percentages do not sum to 100 as categories are not mutually exclusive

represents an inclusion rate of $26 \%$ and $34 \%$, respectively. Using the Pragmatic-Explanatory Continuum Indicator Summary (PRECIS-2) toolkit, a tool designed to measure where a trial falls on the pragmatic versus explanatory continuum on several domains, the setting of the Aqueous-PREP and PREPARE trials received a score of 4 out of a possible 5 indicating that the trials are "mostly pragmatic." This score is reflective of the fact that most of our included orthopedic practices are affiliated with teaching hospitals that are level I trauma centers and regional referral centers versus a mix of trauma centers and local community hospitals. This is unsurprising as large academic hospitals tend to have both the infrastructure and patient volume to make participation in the PREP-IT trials feasible, whereas smaller community hospitals may have insufficient research infrastructure.

Despite our rigorous cluster selection process, we withdrew one cluster as they were unable to comply with the Aqueous-PREP protocol [6]. Specifically, this cluster was unable to meet the a priori threshold $90 \%$ of patients receiving the allocated antiseptic preparation solution. Additionally, the clinical site was unable to complete the case report forms, which led to incomplete data submission. These issues were the result of changes in research infrastructure from the time of site selection to site initiation. The Executive Committee, with support from the Methodology Core, made the decision to withdraw this site. As the criteria for cluster withdrawal were established prior to the initiation of the trial and a small proportion of data are affected, bias should not be introduced as a result of the decision to withdraw this cluster. Additionally, withdrawing this cluster will improve the overall integrity of the trial, as allowing them to continue in Aqueous-PREP would have led to increased protocol violations, treatment contaminations, and missing data.

As the primary outcome for both the AqueousPREP and PREPARE trials is SSI, we documented infection control processes. To remain pragmatic with data collection, we documented the infection control processes at three different levels: cluster level, surgeon level, and patient level. The most important SSI prevention strategies and infection strategies that are likely to vary from patient-to-patient, including antibiotic prophylaxis and blood sugar control, are documented at the patient level on the case report forms. Other infection control details (e.g., hair removal 


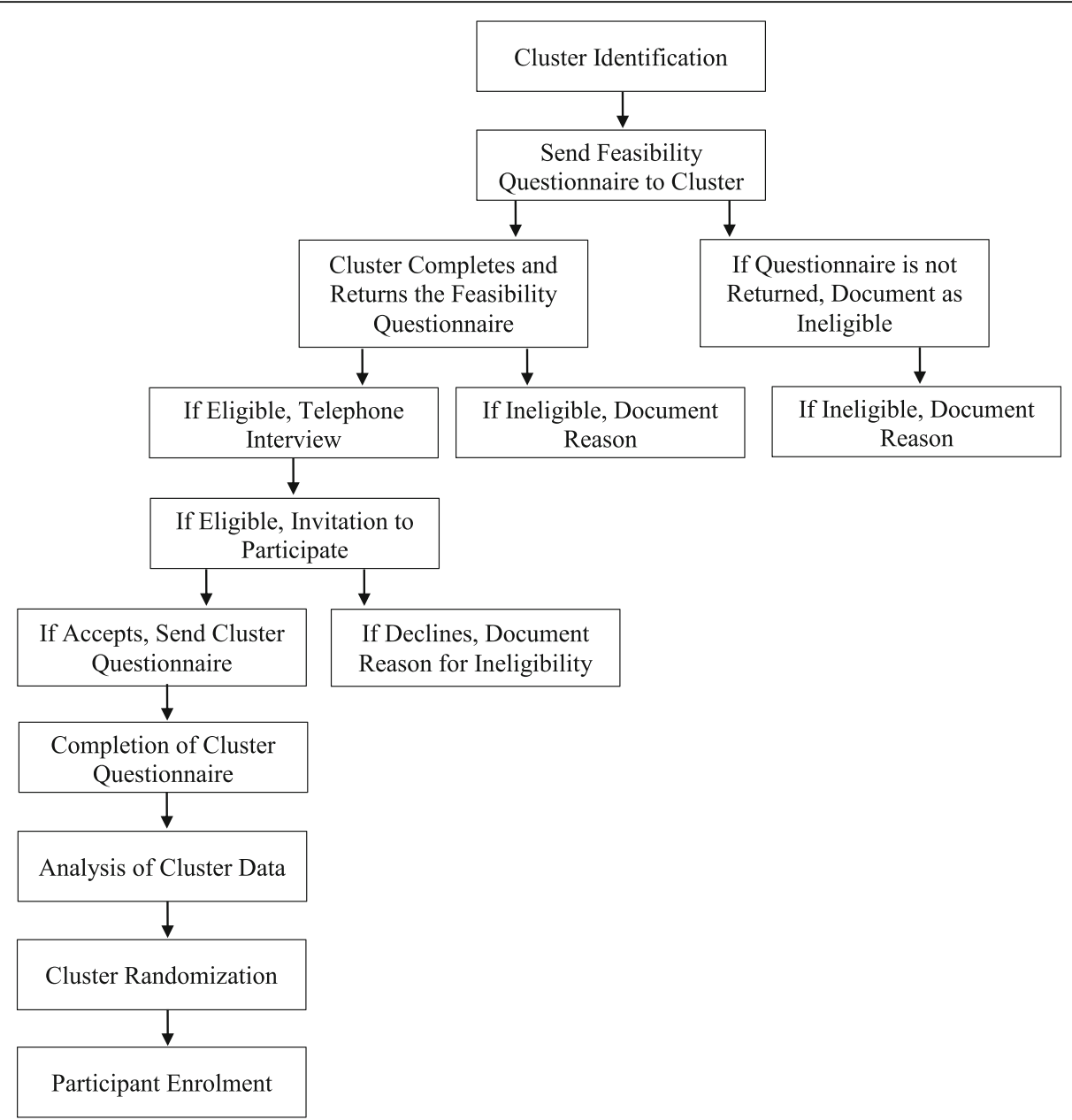

Fig. 4 Overview of the PREP-IT cluster identification, selection, and randomization processes

methods, type of surgical gown) are collected at the surgeon level, as these typically vary by surgeon but remain consistent across patients. Infection control measures that are implemented at the hospital level, including normothermia and decolonization protocols, are documented at the cluster level.

We aimed to select orthopedic practices that follow infection prevention protocols that are representative of the usual care that fracture patients would receive in the real world. We have found some variation in the infection control processes across the clusters in both the Aqueous-PREP trial and the PREPARE trial. Specifically, we found that $64 \%$ of all orthopedic practices in the Aqueous-PPREP trial perform active surveillance cultures for MRSA (64\%). In the PREPARE trial, we found that only $20 \%$ of orthopedic practices perform active surveillance for MRSA in open fracture patients and $15 \%$ for closed fracture patients. In both trials, there was substantial variation in the use of decolonization protocols for MRSA (protocols in use at $64 \%$ of Aqueous-PREP practices and $60 \%$ of PREPARE practices).

Providing the knowledge user with a high level of detail regarding the hospital characteristics, orthopedic surgery details, and infection prevention protocols and procedures allows the knowledge user to determine the generalizability of the trial and the applicability to their hospital and surgical practice.

\section{Conclusions}

When undertaking CRXO trials, it is imperative to follow rigorous reporting standards, as outlined in the CONSORT extensions for CRTs and CRXO trials, including providing knowledge users with a clear definition of the cluster unit, a thorough description of the cluster identification and selection process, and sufficient description of key cluster characteristics. The PREP-IT trials illustrate how this can be accomplished using two large infection prevention CRXO trials in orthopedic fracture patients as an example. 


\section{Appendix}

\section{Characteristics of the withdrawn cluster}

Table 5 Cluster characteristics

\begin{tabular}{ll}
\hline Characteristic & Withdrawn cluster \\
\hline $\begin{array}{ll}\text { Level of trauma care provided } \\
\text { Ownership of hospital }\end{array}$ & $\begin{array}{l}\text { Level I } \\
\text { order affiliated }\end{array}$ \\
Hospital affiliation & $\begin{array}{l}\text { Hospital system, } \\
\text { free-standing }\end{array}$ \\
Population size served & $6,000,000$ \\
Number of inpatient beds & 640 \\
Number of operating rooms & 17 \\
Number of beds in intensive care unit & 102 \\
Hospital is a primary teaching hospital for & Yes \\
a medical school & \\
\hline
\end{tabular}

Table 6 Surgical infection prevention information

\begin{tabular}{|c|c|}
\hline Characteristic & Withdrawn cluster \\
\hline Number of infection preventionists per 250 hospital beds & 5 \\
\hline Infection preventionists certified in infection control & 1 \\
\hline Perform active surveillance cultures for MRSA in open fracture patients & No \\
\hline Perform active surveillance cultures for VRE in open fracture patients & No \\
\hline $\begin{array}{l}\text { Perform active surveillance cultures for other organisms in patients with } \\
\text { open fractures }\end{array}$ & No \\
\hline Decolonization protocol for MRSA & Yes \\
\hline $\begin{array}{l}\text { Policies and/or guidelines on maintaining normothermia during } \\
\text { perioperative period }\end{array}$ & Yes \\
\hline Airflow system(s) in operating room & Conventional ventilation \\
\hline \multirow[t]{4}{*}{ Parameters in place in hospital operating rooms } & High-efficiency particulate air (HEPA) filters \\
\hline & Operating rooms are positive pressure \\
\hline & Air changes per hour \\
\hline & $\begin{array}{l}\text { Temperature maintained between } 20 \text { and } 24^{\circ} \mathrm{C} \text { and humidity between } \\
2 \text { and } 60 \%\end{array}$ \\
\hline
\end{tabular}




\section{Supplementary information}

Supplementary information accompanies this paper at https://doi.org/10. 1186/s13063-020-04611-9.

Additional file 1. THE PREP-IT Investigators.

\section{Abbreviations}

PREP-IT Program: The Program of Randomized trials to Evaluate Pre-operative antiseptic skin solutions in orthopaedic Trauma; RCTs: Randomized controlled trials; CRXO: Cluster randomized crossover; SSI: Surgical site infection; Aqueous-PREP: A Pragmatic Randomized trial Evaluating Pre-operative aqueous antiseptic skin solutions in open fractures; PREPARE: A Pragmatic Randomized trial Evaluating Pre-operative Alcohol skin solutions in Fractured Extremities; CDC: Center for Disease Control and Prevention; IQR: Interquartile range; MRSA: Methicillin-resistant Staphylococcus aureus; VRE: Vancomycin-resistant Enterococcus; HEPA: High-efficiency particulate air; PRECIS-2: Pragmatic-Explanatory Continuum Indicator Summary

\section{Acknowledgements}

We acknowledge and appreciate the many patients, nurses, national organizations, and others who have contributed to the success of this trial. Additional file 1 details the names, affiliations, and roles of the PREP-IT team; the Executive Committee is responsible for the overall conduct of the trial and is comprised of the Principal Investigators and a patient partner. The Executive Committee is advised by a Steering Committee; multiple clinical, research, and stakeholder specialty cores; and experts in patient engagement (University of Maryland PATIENTS Program). An Adjudication Committee reviews participant eligibility and reported study events. The Methods Centre is responsible for the day-to-day management of the PREP-IT trials, which includes clinical site management, data management, and data analysis. The Administrative Centre is responsible for piloting each trial protocol, contracting with each clinical site, and overseeing the Central Institutional Review Board activities.

\section{Authors' contributions}

SS, MB, and GPS conceived the study. SS, TS, and LT analyzed and interpreted the data. SS, TS, SD, DP, and PM drafted the manuscript. All authors critically revised the manuscript and gave final approval of the version to be published.

\section{Funding}

The PREPARE trial is funded by the Patient-Centered Outcomes Research Institute (PCORI) (PCS-1609-36512) and the Canadian Institutes of Health Research (CIHR) (Foundation Grant); the Aqueous-PREP trial is funded by the US Department of Defense (W81XWH-17-1-070) and the CIHR (Foundation Grant). McMaster University Surgical Associates funded start-up activities at the Methods Centre and The Physician Services Incorporated provided funding to the Methods Centre and Hamilton Health Sciences for the AqueousPREP trial. The views in this publication are solely the responsibility of the authors and do not necessarily represent the views of the PCORI, its board of governors, or methodology committee.

\section{Availability of data and materials}

The datasets used and/or analyzed during the current study are available from the corresponding author on reasonable request.

\section{Ethics approval and consent to participate}

Ethics approval for PREPARE and A-PREP was obtained through the Hamilton Integrated Research Ethics Board (Project \#4913 and \#4336) and Advarra (Pro00028360 and Pro00023709). Informed consent was obtained for all study participants enrolled in the PREP-IT trials.

\section{Consent for publication}

Not applicable.

\section{Competing interests}

The authors declare that they have no competing interests.

\section{Author details}

'Department of Surgery, Division of Orthopaedic Surgery, McMaster University, 293 Wellington Street North, Suite 110, Hamilton, ON L8L 8E7, Canada. ${ }^{2}$ Department of Health Research Methods, Evidence, and Impact, McMaster University, 293 Wellington St. N., Suite 110, Hamilton, ON L8L 8E7, Canada. ${ }^{3}$ Department of Epidemiology and Public Health, University of Maryland School of Medicine, Baltimore, MD, USA. ${ }^{4}$ Association of periOperative Registered Nurses, Denver, CO, USA. ${ }^{5}$ Department of Orthopaedic Surgery, University of Pennsylvania Perelman School of Medicine, Philadelphia, PA, USA. ${ }^{6}$ Department of Orthopaedic Surgery, Indiana University School of Medicine, Indianapolis, IN, USA. ${ }^{7}$ Department of Orthopaedics, Banner Health and the University of Arizona-Tucson, Tucson, AZ, USA. ${ }^{8}$ Orthopedic Department, Parc Taulí Hospital Universitari, Institut d'Investigació i Innovació Parc Taulí I3PT, Universitat Autònoma de Barcelona, Sabadell, Spain. ${ }^{9}$ Department of Orthopaedic Surgery and Traumatology, University Hospital Vall d'Hebron, Barcelona, Spain. ${ }^{10}$ Department of Medicine, University of Maryland School of Medicine, Baltimore, MD, USA.

${ }^{11}$ Department of Orthopaedics, University of Maryland, R Adams Cowley Shock Trauma Center, Baltimore, MD, USA.

Received: 6 April 2020 Accepted: 15 July 2020

Published online: 12 August 2020

\section{References}

1. Sackett D. Rules of evidence and clinical recommendations on the use of antithrombotic agents. Chest. 1989:95(2 Suppl):2S-4S.

2. Arnup SJ, Forbes AB, Kahan BC, Morgan KE, McDonald S, McKenzie JE. The use of the cluster randomized crossover design in clinical trials: protocol for a systematic review. Syst Rev. 2014. https://doi.org/10.1186/2046-4053-3-86.

3. Arnup SJ, McKenzie JE, Hemming K, Pilcher D, Forbes AB. Understanding the cluster randomised crossover design: a graphical illustraton of the components of variation and a sample size tutorial. Trials. 2017. https://doi. org/10.1186/s13063-017-2113-2.

4. Campbell MK, Piaggio G, Elbourne DR, Altman DG. CONSORT 2010 statement: extension to cluster randomised trials. BMJ. 2012;345(sep04 1): e5661. https://doi.org/10.1136/bmj.e5661.

5. Arnup SJ, Forbes AB, Kahan BC, Morgan KE, McKenzie JE. The quality of reporting in cluster randomised crossover trials: proposal for reporting items and an assessment of reporting quality. Trials. 2016. https://doi.org/10.1186/ s13063-016-1685-6.

6. Program of Randomized Trials to Evaluate Pre-operative Antiseptic Skin Solutions in Orthopaedic Trauma (PREP-IT) Investigators, Slobogean GP, Sprague S, Wells J, Bhandari M, Rojas A, Garibaldi A, Wood A, Howe A, Harris AD, Petrisor BA, Mullins DC, Pogorzelski D, Marvel D, Heels-Ansdell D, Mossuto F, Grissom F, Del Fabbro G, Guyatt GH, Della Rocca GJ, Nguyen U, et al. Effectiveness of iodophor vs chlorhexidine solutions for surgical site infections and unplanned reoperations for patients who underwent fracture repair: the PREP-IT master protocol. JAMA Network Open. 2020;3(4):e202215. https://doi.org/10.1001/jamanetworkopen.2020.2215.

7. Centers for Disease Control and Prevention (CDC). Surgical site infection (SSI) event.; 2017.

8. Ford I, Norrie J. Pragmatic trials. N Engl J Med. 2016. https://doi.org/10.1056/ NEJMra1510059.

\section{Publisher's Note}

Springer Nature remains neutral with regard to jurisdictional claims in published maps and institutional affiliations.

Ready to submit your research? Choose BMC and benefit from:

- fast, convenient online submission

- thorough peer review by experienced researchers in your field

- rapid publication on acceptance

- support for research data, including large and complex data types

- gold Open Access which fosters wider collaboration and increased citations

- maximum visibility for your research: over $100 \mathrm{M}$ website views per year

At BMC, research is always in progress.

Learn more biomedcentral.com/submissions 